\title{
Control de proyectos de software: actualidad y retos para la industria cubana
}

\author{
Control of software projects: actuality and challenges for the Cuban industry \\ Jacqueline Marín Sánchez ${ }^{1} \quad$ José Alejandro Lugo García² \\ Recibido 26 de agosto de 2014, aceptado 9 de abril de 2015 \\ Received: August 26, $2014 \quad$ Accepted: April 9, 2015
}

\begin{abstract}
RESUMEN
El proceso de monitoreo y control de proyectos de software es indispensable para conocer el estado del desempeño y progreso del proyecto. La industria cubana de software está llamada a convertirse en una fuente de ingresos confiable para el país. Sin embargo, por lo general, las organizaciones cubanas tienen una escasa cultura en cuanto al monitoreo y control de proyectos que facilite la toma de decisiones, afectando los procesos de desarrollo. Para erradicar estas deficiencias la industria debe lograr la estandarización y calidad en sus procesos, a través de la aplicación de buenas prácticas propuestas para esta área por los modelos y estándares de mejora de procesos de mayor aceptación, así como por reconocidos autores. El presente trabajo expone las características del proceso de monitoreo y control de proyectos de software, actualidad y retos para la industria cubana. El contenido sirve de apoyo para la implantación de un proceso de monitoreo y control de proyectos en esta industria, que utilice una herramienta informática para la gestión de proyectos, lo cual contribuirá a mejorar los procesos de desarrollo actuales.
\end{abstract}

Palabras clave: Monitoreo y control de proyectos, modelos y estándares de mejora de procesos, industria cubana del software.

\section{ABSTRACT}

The process of monitoring and controlling of software projects is essential to know the performance status and project progress. The Cuban software industry is set to become a reliable source of income for the country. However, generally, the Cuban organizations have little culture in terms of monitoring and controlling of projects that facilitate decision-making, affecting the development processes. To eliminate these deficiencies the industry must achieve standardization and quality into their processes, through the application of good practices proposed for this area by the models and standards of process improvement with greater acceptance, as well as renowned authors. The present study describes the characteristics of the process for monitoring and controlling of software projects, current affairs and challenges for the Cuban industry. The content provides support for the implementation of a process for monitoring and controlling of projects into this industry, that uses an IT project management tool, which will help to improve current development processes.

Keywords: Monitoring and control of projects, models and process improvement standards, Cuban software industry.

\footnotetext{
1 Centro de Informática Médica. Universidad de las Ciencias Informáticas. Carretera a San Antonio de los Baños, km 2 1⁄2, Torrens, La Lisa. La Habana, Cuba. CP.: 19370. E-mail: jmarin@uci.cu

2 Laboratorio de Investigaciones en Gestión de Proyectos. Universidad de las Ciencias Informáticas. Carretera a San Antonio de los Baños, km 2 플, Torrens, La Lisa. La Habana, Cuba. CP.: 19370. E-mail: jalugo@uci.cu
} 


\section{INTRODUCCIÓN}

El desarrollo de software organizado en proyectos proporciona estabilidad, control y organización a una actividad que puede, si no se controla, volverse caótica [1]. El proceso de monitoreo y control de proyectos de software es indispensable para comprender el progreso del proyecto [2], a través del análisis de indicadores, obtenidos de la recolección de datos del desempeño durante su ejecución, facilitando así la toma de decisiones [3].

Existen modelos y estándares de mejora de procesos que proporcionan directrices, reglas y métodos para el entendimiento del monitoreo y control de un proyecto. El uso de estos está cobrando cada vez más fuerza en las organizaciones, donde uno de los mayores beneficios es la armonización de las diferentes terminologías y el conocimiento de los procesos productivos y los métodos, además de incidir en la mejora continua y la satisfacción de los clientes. Sin embargo, es importante señalar el papel meramente teórico que ejercen los mismos, orientados a la didáctica y a las certificaciones, siendo las entidades las encargadas de definir sus metodologías de trabajo, así como las técnicas y herramientas [3-4].

Las tareas requeridas para lograr los objetivos de la gestión de proyectos no se deberían realizar manualmente ${ }^{3}[1]$. Dada la creciente complejidad para manejar la información resultante del monitoreo y control de proyectos, se muestra evidente la necesidad de la utilización de herramientas informáticas que incluyan técnicas de comportamiento, evaluación y pronósticos y permita conocer el desempeño de los proyectos, según las estructuras o niveles funcionales de la organización [5].

Los recientes Lineamientos de la Política Económica y Social del Partido Comunista de Cuba hacen un llamado a incrementar la soberanía tecnológica y la organización, fortaleciendo los procesos de monitoreo y control sistemático, con el fin de lograr resultados esperados de acuerdo a los planes establecidos con eficiencia, orden y disciplina [6]. En el escenario actual, la industria nacional del software presenta deficiencias en la organización, estandarización de

3 Refiriéndose a utilizar un Sistema de Información para la Gestión de Proyectos (PMIS) manual (papel y lápiz). sus procesos y normalización de la calidad, elementos que están afectando su potencialidad [7]. Para esto, el desarrollo de software en el país, necesita contar con métodos de mejora y estandarización del proceso de monitoreo y control de proyectos, adaptable a las características y necesidades de las entidades productoras de software, y que favorezca la toma de decisiones en los distintos niveles de dirección.

El presente trabajo tiene como objetivo exponer las características del proceso de monitoreo y control de proyectos de software, su actualidad y retos para la industria cubana en este campo.

\section{ANÁLISIS TEÓRICO}

\section{MONITOREO Y CONTROL DE PROYECTOS DE SOFTWARE}

La mayoría de los profesionales de la gestión de proyectos con experiencia saben que no existe una única manera de gestionar un proyecto. Se aplican los conocimientos de gestión, habilidades y procesos necesarios en un orden de preferencia y con diferente rigor para lograr el desempeño deseado del proyecto [3]. La evidencia sugiere que se está ganando experiencias en el monitoreo y control de proyectos a través de la aplicación de modelos y/o estándares de mejora de procesos [8]. A continuación se presentan las principales características del proceso de monitoreo y control de proyectos sugeridas por los modelos y estándares de mejora de procesos de mayor aceptación internacional.

A Guide to the Project Management Body of Knowledge - PMBOK Guide (América del Norte) Para $\mathrm{PMBOK}^{4}$, el proceso de monitoreo y control de proyectos está compuesto por aquellas actividades requeridas para supervisar, analizar y regular el progreso y el desempeño del proyecto, para identificar áreas en las que el plan requiera cambios y para iniciar los cambios correspondientes. La 5ta edición de esta guía plantea la necesidad de monitorear y controlar el proyecto a través de los datos de desempeño de cada una de las áreas de conocimiento de la gestión de proyectos: Integración, Alcance, Tiempo, Costo, Calidad, Recursos Humanos, Comunicaciones,

4 Desarrollada por el PMI en 1987, la Guía de los Fundamentos para la Dirección de Proyectos (PMBOK por sus siglas en inglés) [3] se publica cada cuatro años y cuenta con cinco ediciones hasta la actualidad. 
Riesgos, Adquisiciones e Interesados. Estos datos se procesan a través del cálculo de indicadores, se analizan en su contexto y se transforman para convertirse en información del proyecto durante varios procesos de monitoreo y control. La información puede entonces ser comunicada verbalmente o almacenada y distribuida como informes en varios formatos.

Esta guía resalta la necesidad de gestionar lecciones aprendidas en cada una de las áreas y manejarlas a través de bases de conocimientos puestas a disposición de la organización. Esto permitirá a los jefes de proyecto contar con datos históricos y experiencias de proyectos similares para la toma de decisiones.

PMBOK constituye una guía suficientemente completa respecto a los objetivos y actividades a desarrollar para monitorear y controlar un proyecto, proponiendo además técnicas y herramientas para llevar a cabo los procesos. Sin embargo, por ser descrita para todo tipo de proyecto y no explicar cómo llevar a cabo sus procesos, suele ser abstracta y genérica, tornando engorrosa su aplicación en entornos reales de proyectos de desarrollo de software. De igual manera sucede con el manejo de los indicadores, el estándar propone las fórmulas de cálculo solo para controlar algunas áreas de conocimiento, describiendo el resto de forma verbal. Esto provoca dudas a la hora de aplicarlos, además de hacerse difícil la manipulación manual de los mismos. Por último, el hecho de que sea una guía dirigida principalmente a los jefes de proyecto, no define el resto de los roles involucrados en el proceso.

\section{Capability Maturity Model Integration for} Development - CMMI-DEV (América del Norte) El propósito del monitoreo y control del proyecto, según $\mathrm{CMMI}^{5}$, es proporcionar una comprensión del progreso del proyecto para que se puedan tomar las acciones correctivas apropiadas, cuando el desempeño del mismo se desvíe significativamente del plan [2]. El plan de proyecto bien definido es la base para la monitorización de las actividades, la comunicación del estado y la toma de acciones correctivas. El

\footnotetext{
5 El Modelo de Madurez y Capacidad Integrado para Desarrollo (CMMI-DEV por sus siglas en inglés), desarrollado por el SEI, constituye una colección de mejores prácticas para ayudar a las organizaciones dedicadas al desarrollo de productos y servicios de software, en la mejora y evaluación de sus procesos.
}

progreso se determina principalmente comparando los atributos de los productos de trabajo y de las tareas, el esfuerzo, los compromisos, los datos, los riesgos, la involucración de los interesados, el coste y el cronograma reales, con el plan en los hitos o niveles de control establecidos en el plan del proyecto o en la estructura de desglose del trabajo (WBS).

El modelo propone llevar a cabo acciones correctivas según proceda cuando el estado real del proyecto se desvíe significativamente de los valores esperados. Teniendo en cuenta que una desviación es significativa si, cuando se deja sin resolver, impide al proyecto cumplir con sus objetivos. Las acciones correctivas pueden requerir replanificación del cronograma, la inclusión de nuevas tareas y/o el establecimiento de nuevos acuerdos y un seguimiento sistemático con el fin de verificar el cumplimiento de las mismas. Las lecciones aprendidas como resultado de tomar acciones correctivas pueden ser entradas a los procesos de planificación y de gestión de riesgos.

CMMI hace alusión al establecimiento de métricas (de las cuales son derivables indicadores) y cómo éstas se pueden utilizar para monitorear, controlar y evaluar el desempeño del proyecto de software. No obstante la descripción de las mismas es verbal, no define indicadores específicos y/o métodos concretos para la evaluación de la ejecución de proyectos a partir de indicadores.

\section{Normas ISO (EUROPA)}

La Organización Internacional de Normalización [9], es el organismo no gubernamental encargado de promover el desarrollo de normas internacionales de fabricación (tanto de productos como de servicios), comercio y comunicación para todas las ramas industriales a excepción de la eléctrica y la electrónica. La ISO, junto a la Comisión Electrotécnica Internacional (IEC por sus siglas en Inglés) [10], forma el sistema especializado para la normalización mundial ISO/IEC.

\section{ISO 10006:2007}

La ISO 10006:2007 ${ }^{6}$ [11] propone monitorear y controlar, a lo largo de todo el proyecto,

\footnotetext{
6 La ISO 10006:2007 lleva por título "Sistemas de gestión de la calidad-Directrices para la gestión de la calidad en los proyectos" y tiene como objetivo proponer elementos, conceptos y prácticas para la gestión de la calidad en la gestión de proyectos.
} 
las actividades, cronograma, costos, riesgos, comunicaciones y el contrato, con el objetivo de evaluar periódicamente el desempeño y avance e identificar posibles deficiencias y oportunidades de mejora. Los datos e información derivados de estas evaluaciones deben ser analizados para identificar tendencias o posibles incertidumbres en el trabajo restante del proyecto y de esta forma tomar decisiones eficaces. Para asegurar un monitoreo y control adecuado del proyecto se deben establecer los tiempos de revisión del cronograma; definir indicadores del desempeño, la forma de medirlos y la frecuencia de recopilación de los datos; confirmar que los objetivos del proyecto continúan siendo válidos en un entorno cambiante para el proyecto y establecer acciones correctivas y/o preventivas en caso de ser necesario.

\section{ISO/IEC 12207:2008}

Para la ISO/IEC 12207:20087 [12] el objetivo del proceso de monitoreo y control de proyectos es determinar el estado del proyecto y asegurar que el mismo se realice de acuerdo a los planes y cronogramas, dentro de los presupuestos proyectados, y que se satisfagan los objetivos técnicos. Para ello es necesario supervisar la ejecución general del proyecto, proporcionando informes del avance que permitan analizar y resolver los problemas detectados durante la misma.

ISO 12207:2008 pone especial énfasis en la gestión de las decisiones con el fin de seleccionar las alternativas y acciones más beneficiosas y óptimas para el proyecto. Este último debe definir una estrategia de toma de decisiones que incluya la identificación de categorías para las decisiones, un esquema de asignación de prioridades y la identificación de los responsables. Las decisiones pueden surgir como resultado de una evaluación de la eficacia, una compensación técnica, un problema que necesita ser resuelto, acción necesaria como respuesta al riesgo de exceder el tiempo aceptable, y/o la aprobación del proyecto a la siguiente etapa del ciclo de vida. Los resultados de las decisiones se deben registrar, controlar, evaluar e informar para confirmar que los problemas se han resuelto

\footnotetext{
7 La ISO/IEC 12207:2008 es el estándar que propone un marco de trabajo común para los procesos de ciclo de vida del software y se denomina "Sistemas e ingeniería de software - Procesos del ciclo de vida del software".
}

de manera eficaz, las tendencias adversas se han invertido y se han aprovechado las oportunidades.

Las normas ISO no proponen técnicas y herramientas ni ejemplos para llevar a cabo los procesos. Consisten en estándares abiertos con explicaciones sencillas sobre qué se debería hacer y no muestran la forma de cumplir con sus requisitos. Pese a esto, la orientación a procesos, el enfoque al cliente, la necesidad de tomar decisiones eficaces y la propuesta de mejora continua como bases fundamentales de las normas, las convierten en mejores prácticas a tener en cuenta.

\section{ESTADO DEL MONITOREO Y CONTROL DE PROYECTOS DE SOFTWARE EN CUBA}

El desarrollo de software en Cuba, está llamado a convertirse en una representativa fuente de ingresos para el país [13]. Para esto, según palabras del Viceministro de la Informática y las Comunicaciones, las organizaciones necesitan dotarse de herramientas adecuadas que contribuyan a incrementar la calidad durante los procesos de desarrollo de software que se ejecutan, en aras de fortalecer la industria [14]. Entrevistas realizadas por los autores a varios especialistas como [5, 15-17], reflejan que algunos de los principales problemas del desarrollo de software en el país vienen dados por insuficiencias durante el proceso de monitoreo y control de los proyectos, entre ellos resaltan:

- Escasa cultura en las prácticas de monitoreo y control.

- Iniciativas aisladas en función de mejorar este proceso.

- La supervisión se realiza, en la mayoría de los casos, en base a preguntas que los jefes realizan a sus subordinados y no en función del análisis de indicadores objetivos, que caractericen y faciliten la evaluación del desempeño de los proyectos.

- Toma de decisiones basadas en elementos subjetivos, resultando impredecible el progreso del proyecto.

- Insuficiente uso de herramientas de apoyo al proceso.

- Escaso uso de bases de conocimiento que permitan la gestión de lecciones aprendidas durante el proceso. 
Pese a estas deficiencias, encuestas realizadas durante la XV Convención y Feria Internacional Informática 2013 a 21 entidades del sector [18], evidencian que existe una voluntad organizacional hacia el ordenamiento de sus procesos (el 100\% de las organizaciones desarrolla basado en procesos) a través de la aplicación de buenas prácticas establecidas por modelos y estándares de reconocimiento internacional: el 14\% afirma usar prácticas descritas por CMMI; un $36 \%$ se guía por ISO 9001; un $14 \%$ usa un híbrido entre estas dos y otras de menor reconocimiento, aunque solo se aplican estas prácticas de forma parcial y no para todas las áreas; y un 36\% no se rige por algún modelo o estándar. Aun así, es escasa la documentación publicada acerca de los resultados obtenidos del esfuerzo en función de estandarizar los procesos en la industria.

Una experiencia pionera en la mejora de procesos en Cuba lo constituye la certificación del nivel 2 de CMMI-DEV en 3 centros de desarrollo de la Universidad de las Ciencias Informáticas (UCI), durante el año 2011. Como parte del Programa de Mejora de Procesos llevado a cabo en la UCI, se implantó el proceso Monitoreo y Control de Proyecto (PMC) que responde al área de igual nombre del modelo. PMC [19] está compuesto por un único subproceso que se estará ejecutando constantemente a lo largo del ciclo de vida del proyecto hasta el cierre del mismo [20].

Dada la creciente complejidad para manejar la información resultante del monitoreo y control de proyectos, se muestra evidente la necesidad de la utilización de herramientas tecnológicas que incrementen los niveles de automatización como soporte al proceso. Una numerosa cantidad de herramientas informáticas para la gestión de proyectos han sido creadas en las últimas décadas [21]. Aunque muchas satisfacen las necesidades de sus clientes, no siempre cubren las expectativas de todos por temas tan específicos o combinados como precio, tipo de licencia (privativa o de software libre o código abierto), soporte o insuficiencias en el manejo de ciertos datos. En los últimos cinco años, iniciativas cubanas han realizado aportes al desarrollo de herramientas de este tipo ante la necesidad de personalizacion de la industria.

Desarrollada por el Laboratorio de Investigaciones en Gestión de Proyectos de la UCI, la Suite de
Gestión de Proyectos Xedro GESPRO [22], ha sido desplegada en esta institución como soporte a su Programa de Mejora de Procesos. Construido sobre software de código abierto y herramientas de la propia organización, el sistema basa su funcionamiento en la implementación de buenas prácticas sugeridas por los estándares de mejora de procesos PMBOK y CMMI, permitiendo la gestión de las áreas de conocimiento y procesos asociados con dichos estándares. En su última versión (14.05), GESPRO incluye un Cuadro de Mando Integral (CMI) que contribuye al control tanto de portafolios de proyectos como proyectos independientes. Cuenta además, con un conjunto de indicadores clave que facilitan conocer el estado del desempeño del proyecto y la organización respecto a áreas fundamentales de la gestión como: rendimiento de la ejecución (IRE), rendimiento de la planificación (IRP), rendimiento de los costos (IRC), la eficacia (IREF), la logística (IRL), los recursos humanos (IRHH) y la calidad de los datos (ICD) [23]. Igualmente, incluye reportes predeterminados y la posibilidad de añadir otros según las necesidades específicas del entorno. La información asociada con el estado de los proyectos en cada corte (indicadores y evaluación del proyecto), se almacena en una base de conocimiento de proyectos terminados con el fin de aplicar posteriores mecanismos de aprendizaje automático sobre los datos [24-25].

Otra iniciativa cubana de herramienta tecnológica de apoyo al control de proyectos de software, es la desarrollada por el Complejo de Investigaciones Tecnológicas Integradas (CITI) [26]. La propuesta se vale de un conjunto de sistemas informáticos integrados que permiten el monitoreo y control de proyectos y recursos humanos, a través de un tablero de control que proporciona diferentes vistas de reportes e indicadores como IRP, IRC y métricas específicas del desarrollo en la institución. La solución está implementada sobre las tecnologías: TFS y SharePoint, además de un portal web desarrollado sobre .NET.

\section{RESULTADOS}

\section{RETOS PARA LA INDUSTRIA CUBANA DEL SOFTWARE}

Las características actuales del desarrollo de software en el país, tornan difícil el financiamiento 
e implantación de estándares internacionales de referencia para las organizaciones de esta rama. Atendiendo a [7, 18, 27], la industria cubana del software está conformada principalmente por pequeñas y medianas organizaciones o pymes como se conoce comúnmente, compuestas generalmente por personal joven y no necesariamente especializado en mejora de procesos. Esto se traduce, considerando las investigaciones realizadas por [28-29], en escasez de tiempo, recursos (tanto financieros como humanos) y capacitación para lograr la implantación de estos estándares foráneos.

Evidencia de lo anterior lo consituye el Programa de Mejora de la UCI, durante el cual se consumió, para la definición de los procesos que responden a las siete áreas del nivel dos de CMMI, 17356 horas, lo que equivale a 723 días de trabajo, con un esfuerzo de 810 horas/hombre. De dicho esfuerzo, 135 horas/ hombres fue dedicado al proceso de Monitoreo y Control de Proyectos (PMC), aproximadamente el 17\%. Agregando a estas cifras un total de 442 horas dedicadas a la capacitación de los proyectos sobre los procesos, de las cuales 76 fueron dedicadas a PMC (un 17\%), constituyendo el área de mayor dedicación [20].

En el análisis de los datos mencionados, no se considera el esfuerzo dedicado a capacitar al personal que definió los procesos; el destinado a que los directivos aprobaran los elementos del programa; el trabajo realizado inicialmente en los proyectos pilotos y posteriormente en el resto de los proyectos de cada centro; las revisiones a los mismos; la preparación del SCAMPI (del inglés Standard CMMI Appraisal Method for Process Improvement) interno y externo. A estas cifras debe sumarse el costo monetario de una consultoría especializada y el pago de la evaluación final que superan los 70000 USD [18].

Con el fin de consolidar la industria, el Ministerio de Comunicaciones (MINCOM) lleva a cabo el Programa "Desarrollo de la industria cubana del software". El mismo está dirigido hacia retos y objetivos como: identificar los modelos organizativos y marcos de trabajo que debe tener la industria de software para las condiciones de Cuba; fortalecer la seguridad y soberanía tecnológica en la producción de software y la prestación de servicios informáticos con elevado valor agregado; y potenciar el desarrollo sostenible de distribuciones cubanas de software sobre plataformas de código abierto, que respondan a las necesidades actuales de la nación [30].

En respuesta a estos objetivos el MINCOM decide apostar por el diseño de un modelo cubano para el desarrollo de las aplicaciones informáticas que se implementan en las organizaciones del país. El mismo cuenta, por la importancia que se le atribuye al área, con un proceso base para gestión de proyectos que enfoca algunas de sus directrices en el monitoreo y control de proyectos. Sin embargo, el mencionado modelo aún no está liberado para su uso en la industria [7].

La necesidad de mantener un monitoreo y control sistemático de los proyectos debe partir desde la propia dirección estratégica de la organización. Entendiendo por estrategia al plan detallado que establece la dirección y que guía la utilización de recursos para alcanzar la visión de la organización, misión, dimensiones (estratégica, táctica, operativa) y objetivos estratégicos. La organización debe ser capaz de ganar madurez desde un enfoque de dirección orientada a proyectos y viceversa [31].

Con el objetivo de llevar a cabo un proceso de monitoreo y control adecuado en la industria cubana del software, es necesario partir de una buena planificación de los proyectos. Para ello, según Delgado Victore [5], uno de los aspectos más importantes es la determinación de la línea base del proyecto con la información inicial del mismo. La línea base cuenta con la información de referencia del proyecto utilizada para monitorearlo y controlarlo y de esta forma comparar su avance. La línea base como contenido dispone de la fecha de inicio, fin, los recursos planificados que intervienen en cada una de las tareas, duración de las mismas y el costo entre otros indicadores que se llevan de forma controlada, para comparar los ajustes intermedios con la programación inicial y medir las desviaciones del proyecto con relación a sus objetivos.

Cuanto más cuantitativo sea el enfoque para comprobar el cumplimiento de la línea base, se estará probablemente más preparado para prevenir problemas potenciales y responder ante ellos de un modo proactivo [1]. En función de esto Kaplan y Norton [32] afirman que "Las mediciones son importantes: si no puedes medirlo, no puedes 
gestionarlo". Para ello es necesaria la implementación de un CMI que transforme los objetivos en indicadores de desempeño y proporcione un marco de trabajo, una estructura y un lenguaje para comunicar el estado y progreso del proyecto y la estrategia a seguir. El CMI debe ser utilizado como un sistema de comunicación, de información y de formación, y no como un sistema de control.

Como estrategia a seguir, Delgado Victore [5] sugiere que los indicadores de desempeño sean monitoreados y controlados por cortes planificados en el proyecto, como muestra la Figura 1. La información que se genera en cada corte (estado real del proyecto con relación a la línea base previamente establecida) permite tomar decisiones estratégicas, garantizando el cumplimiento de los objetivos del proyecto.

Después de tomadas las decisiones y fijadas las metas para el próximo corte I+1, se debe elaborar un breve informe de estado sobre una plantilla que recoge la información del proyecto, vinculada al hito en el corte I. El informe de estado recoge el análisis del comportamiento, el cumplimiento de los acuerdos en el corte I-1, la caracterización del proyecto en el corte I, los indicadores, las decisiones con sus respectivos acuerdos, la evaluación y las metas para el corte I+1, entre otros aspectos de interés, ver Figura 2. Mediante este informe el jefe de proyecto puede realizar una evaluación integral del proyecto, a partir de un conjunto de indicadores estratégicos ponderados y reflejados en el tablero de comando [5].

La información generada a través del análisis de los indicadores de desempeño debe ser utilizada para la toma de decisiones a nivel de organización, conduciendo a los jefes de proyecto a mantener sus proyectos actualizados y el mismo grado de control que en niveles superiores. El monitoreo y control debe ser dosificado (en cascada), tomando en consideración los datos relevantes por niveles en la toma de decisiones (e. g. organización, proyecto, individuo) [5, 23]. Guido y Clements recomiendan que mientras más corto sea el período de recopilación de información sobre el desempeño del proyecto, o lo que es lo mismo, cortes para monitoreo y control, mayores serán las posibilidades de identificar tempranamente los problemas y llevar a cabo acciones correctivas efectivas [33].
Las tareas requeridas para lograr los objetivos de la gestión de proyectos, no se deberían realizar manualmente (PMIS utilizando papel y lápiz) [1]. Esto requiere de un cambio de mentalidad, un proceso de formación al personal y una disciplina tecnológica motivada y exigida por los directivos de la organización [34].

Un proyecto está compuesto por personas y el éxito está en sus hombros [35]. La puesta en práctica o implementación de cualquier estrategia debe comenzar por la educación e involucramiento de las personas que en ella participan. El jefe de proyecto debe otorgar responsabilidad al equipo de proyecto en la toma de decisiones, haciéndole saber a todos sus miembros que la calidad es importante y que no debe verse comprometida. Cuanto más control se le proporcione al equipo de proyecto para tomar decisiones técnicas y del proceso de desarrollo, aplicar lecciones aprendidas de proyectos anteriores a nuevas situaciones, establecer técnicas que permitan la retroalimentación y solución de problemas, mayores serán los logros, la integración y la motivación del mismo.

El monitoreo y control de proyectos apoyado por las Tecnologías de la Información y las Comunicaciones (TIC) introduce un cambio de estilo de dirección en la interacción entre el equipo de proyecto, los directivos y las partes interesadas. Este proceso debe ir apoyado por el uso de herramientas informáticas que implementen técnicas para el análisis de comportamientos, realizar diagnóstico y establecer pronósticos y tendencias, con el propósito de obtener los elementos necesarios para la toma de decisiones en el marco de una gestión de proyecto eficaz [5].

Para motivar la mejora, las lecciones aprendidas se deben documentar a lo largo del ciclo de vida del proyecto, o como mínimo, durante el cierre del mismo. Estas deben generar información para mejorar la gestión y toma de decisiones de futuros proyectos, quedando archivadas en una base de conocimiento de la organización. Una base de conocimiento almacena información histórica y lecciones aprendidas sobre los resultados de las decisiones y el desempeño de los proyectos. Con el fin de agilizar los procesos de intercambio de información y control, las bases de conocimiento deben contener funcionalidades necesarias para la recolección, organización y 


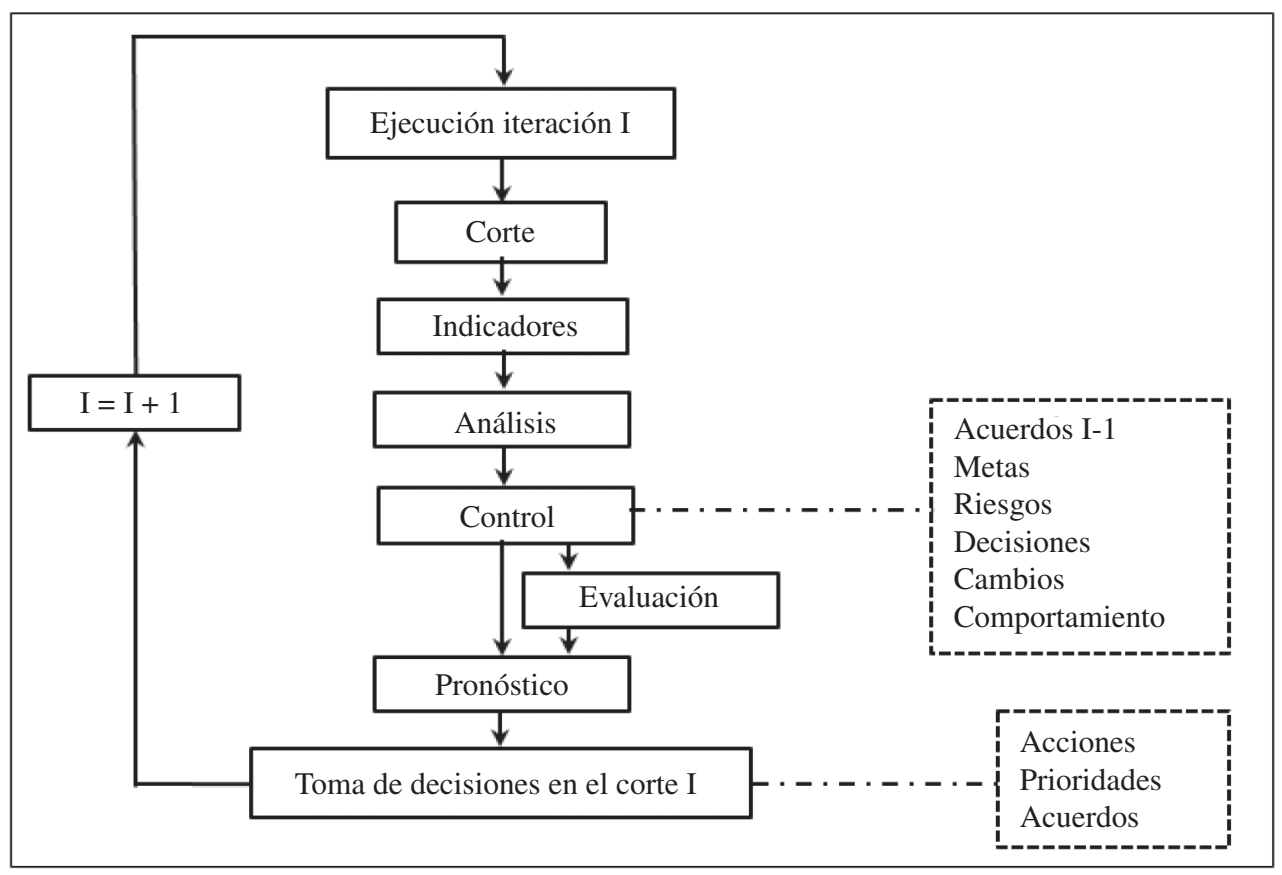

Figura 1. Ciclo para el monitoreo y control de proyectos.

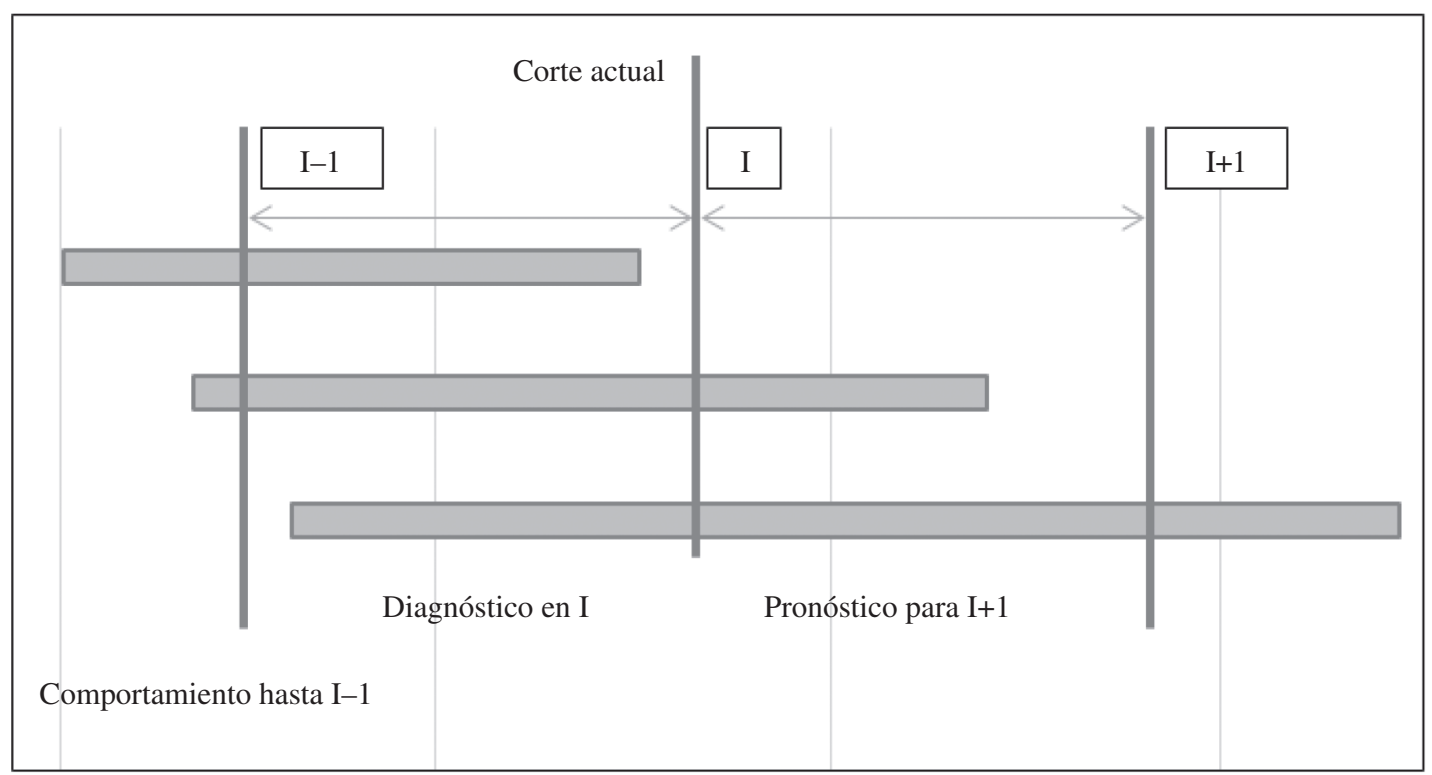

Figura 2. Monitoreo y control por cortes del proyecto.

recuperación de la experiencia acumulada mediante el uso de tecnologías.

A pesar de la existencia de soluciones informáticas nacionales que ya brindan soporte al control de proyectos de software, aún quedan retos a tratar como la estandarización y generalización respecto al uso de las mismas. Todavía se continúa apostando en algunas instituciones por el uso de tecnologías privativas como apoyo al control de proyectos de 
software, situación no acorde con las políticas definidas para el desarrollo de la industria a mediano y largo plazo.

\section{CONCLUSIONES}

La industria cubana del software necesita contar con métodos y herramientas que contribuyan a la estandarización y calidad del proceso de monitoreo y control de proyectos. Las carencias existentes en cuanto a este proceso atentan contra la eficiencia y eficacia del desarrollo de software. Sin embargo, se han realizado esfuerzos en aras de lograr la aplicación de buenas prácticas sugeridas por modelos y estándares de aceptación internacional en esta esfera.

El uso de las TIC como apoyo a los procesos productivos, específicamente al monitoreo y control de proyectos, favorece un control objetivo basado en indicadores y datos históricos que conducen al análisis del comportamiento, estado actual y futuras conductas del proyecto, en función de una toma de decisiones eficiente y eficaz. Para alcanzar la soberanía tecnológica durante el control de los proyectos, es fundamental el uso de herramientas basadas en software de código abierto. El empleo de herramientas informáticas como la Suite de Gestión de Proyectos Xedro GESPRO contribuye al cumplimiento de estos objetivos.

\section{REFERENCIAS}

[1] R.S. Pressman. "Software Engineering: A practitioner's approach". McGraw-Hill. Seventh Edition. New York, EUA. 2010. ISBN: 978-0-07-337597-7.

[2] SEI. "CMMI® for Development, Version 1.3. Improving processes for developing better products and services" Fecha de actualización: Noviembre 2010. Fecha de consulta: 10 de julio 2014. URL: www.sei. cmu.edu/reports/10tr033.pdf

[3] PMI. "A Guide to the Project Management Body of Knowledge. (PMBOK® Guide)". Fifth Edition. Project Management Institute. Pennsylvania, USA Inc. 2013. ISBN: 978-1-935589-67-9.

[4] N. Zabaleta Etxebarria, J. Igartua Lopez y N. Errasti Lozares. "Análisis de la Relación Existente entre los Estándares de Gestión de Proyectos y los Factores Críticos para su Éxito". 6th International Conference on Industrial Engineering and Industrial Management. Vigo, España. 2012.

[5] R. Delgado Victore, J. García Vejerano, A. Delfino Rodríguez, M. Medina Rodríguez, J.L. Muñoz Suarez, E. Hoffmann, M. Ruiz Hernández, Y. M. Almaguer Bajuelo y A. Morales Oliva. "La Dirección Integrada de Proyecto como Centro del Sistema de Control de Gestión en el Ministerio del Poder Popular para la Comunicación y la Información". Caracas, Venezuela. 2011. $N^{\circ}$ Reg. Centro Nacional de Derecho de Autor (CENDA): 07685-7685.

[6] PCC. "Lineamientos de la Política Económica y Social del Partido y la Revolución". Partido Comunista de Cuba. La Habana, Cuba. 2011. Fecha de consulta: 2 de enero 2014. URL: http://www.cubadebate.cu/wp-content/ uploads/2011/05/folleto-lineamientos-vicong.pdf

[7] D. Pérez Montalván. "Guía general para un modelo cubano de desarrollo de aplicaciones informáticas". Tesis para optar al grado de máster. Universidad de las Ciencias Informáticas. La Habana, Cuba. 2014.

[8] P.L. Bannerman. "Barriers to Project Performance". 46th Hawaii International Conference on System Sciences. IEEE Computer Societ, pp. 4324-4333. 2013. ISSN: $1530-1605 / 12$. DOI: $10.1109 /$ HICSS.2013.113.

[9] ISO. "ISO. International Organization for Standardization". Fecha de consulta: 10 de julio 2014. URL: http://www.iso.org/iso/ home.htm.

[10] IEC. "International Electrotechnical Commission". Fecha de consulta: 10 de julio 2014. URL: http://www.iec.ch.

[11] ISO. "NC-ISO 10006:2007. Sistemas de gestión de la calidad-Directrices para la gestión de la calidad en los proyectos (ISO 10006:2003, IDT)". Oficina Nacional de Normalización (NC). 2007.

[12] ISO/IEC. “ISO/IEC 12207:2008(E). Systems and software engineering-Software life cycle processes". 2008.

[13] Y. González. "El proceso de exportación de servicios y su importancia en las condiciones actuales." Fecha de actualización: diciembre 
de 2011. Fecha de consulta: 10 de octubre de 2014. URL: http://www.betsime.disaic. cu/secciones/mer_octdic_11.htm

[14] S. Suzarte Medina. "Instan a garantizar la calidad del software cubano". Fecha de consulta: 10 de julio 2014. URL: http:// www.trabajadores.cu

[15] A.M. García Pérez, N. Aragón González y M. F. Rivero Aragón. "Método para la mejora de procesos empresariales y su informatización". Revista Nueva Empresa. Vol. $9 \mathrm{~N}^{\mathrm{o}} 1$, pp. 54-61. 2013. ISSN: 1682-2455.

[16] M. André Ampuero, M.G. Baldoquín and S.T. Acuña. "Formal model for assigning human resources to teams in software projects". Information and Software Technology. Vol. 53 No 3, pp. 259-275. 2011. ISSN: 0950-5849.

[17] M.R. Abreu Bosch, M.B. Infante Abreu y D. Derivet Thaureaux. "Propuesta para mejorar la productividad de la capacitación en la industria cubana de software". XV Convención y Feria Internacional Informática 2013. La Habana, Cuba. 2013.

[18] K. Ramos Blanco. "Proceso Base de Ingeniería de Requisitos para las pequeñas y medianas empresas de desarrollo de software". Tesis para optar al grado de máster. Universidad de las Ciencias Informáticas. La Habana, Cuba. 2013.

[19] UCI. "IPP-3530_2009 Libro de Proceso Monitoreo y Control de Proyecto". Universidad de las Ciencias Informáticas. La Habana, Cuba. 2009.

[20] K. Ramos Blanco, A. Suárez Batista, D. Pérez Montalván, D. Neuland Agüero, A. Febles Estrada, R. Delgado Martínez y M. Muñoz Roja. "Experiencias del programa de mejora de procesos en la Universidad de las Ciencias Informáticas". Revista Cubana de Ciencias Informáticas. Vol. $5 \mathrm{~N}^{\circ} 2$. AbrilJunio 2011. ISSN: 1994-1536.

[21] J.A. Lugo García. "Modelo para el control de la ejecución de proyectos basado en indicadores y lógica borrosa". Tesis para optar al grado de máster. Universidad de las Ciencias Informáticas. La Habana, Cuba. 2012.

[22] P.Y. Piñero Pérez, S. Torres López, M. Izquierdo Matias, J.A. Lugo García, J. Menéndez Rizo, F.N. Abelardo, A.M. Santiesteban, A.D. Pérez, I. Pérez Pupo,
K.M. Torres, R.M. Pedraza, M. Tase, A. Hernández y R. Delgado Victore. "GESPRO. Paquete para la gestión de proyectos". Revista Nueva Empresa. Vol. $9 \mathrm{~N}^{\circ} 1$, pp. 45-53. 2013. ISSN: 1682-2455.

[23] J.A. Lugo García, S. Torres López, A.M. García Pérez, P. Y. Piñero Pérez y R. Delgado Victore. "Cálculo automático de indicadores como técnica de apoyo para la decisión en el Sistema Nacional de Salud". Revista Cubana de Información en Ciencias de la Salud. Vol. $24 \mathrm{~N}^{\circ}$ 4, pp. 472-481. 2013. ISSN 2307-2113.

[24] I. Pérez Pupo, P.Y. Piñero Pérez, J.A. Lugo García, A.D. Pérez, Y. Piñero y S. Torres López. "Modelo para el aprendizaje automático. Aplicación en la Dirección Integrada de Proyectos". XV Convención y Feria Internacional Informática 2013. La Habana, Cuba. 2013.

[25] A. Bermudez Peña, J.A. Lugo García, I. Pérez Pupo, P.Y. Piñero Pérez y G. Cruz Lemus. "Sistema neuro-borroso de apoyo al control de la ejecución de proyectos". Revista Cubana de Ingeniería. Vol. $5 \mathrm{~N}^{\circ} 2$, pp. 41-51. Agosto 2014. ISSN: 2223-1781.

[26] A. Montero Posada y M. André Ampuero. "Herramienta de soporte a un sistema de métricas e indicadores". Revista Cubana de Ciencias Informáticas. Vol. 7 N² 2, pp. 49-66. 2013. ISSN: 2227-1899.

[27] A. Febles Estrada. "Un modelo de Referencia para la Gestión de Configuración en la PYME de Software". Tesis para optar al grado de doctor. Instituto Superior Politécnico "José Antonio Echeverría”. La Habana, Cuba. 2003.

[28] SELA. "Desarrollo de una Industria Regional de Software en América Latina y el Caribe: Consideraciones y Propuestas". Caracas, Venezuela. 2009. SP/Di N ${ }^{\circ}$ 7-09. Fecha de consulta: 6 de febrero 2015. URL: http:// www.sela.org

[29] OMPI. Organización Mundial de la Propiedad Intelectual. "Información sobre la División de Pequeñas y Medianas Empresas”. Fecha de consulta: 6 de febrero 2015. URL: http:// www.wipo.int/sme/es/about_sme.html.

[30] MINCOM. "Programa: Desarrollo de la Industria Cubana del Software". Fecha de consulta: 5 de febrero 2015. URL: http:// www.mincom.gob.cu/?q=node/321 
[31] G.A. Ronda Pupo. "Dirección Estratégica, constructo y dimensiones". Ediciones Futuro. La Habana, Cuba. 2007. ISBN: 978-959-286-003-2.

[32] R.S. Kaplan y D.P. Norton. "Cuadro de Mando Integral". Gestión 2000. 2da Edición. Barcelona, España. 2002. ISBN: 97884987504085.

[33] J. Gido y J.P. Clements. “Administración Exitosa de Proyectos". [Traducción al español]. Cengage Learning Editores, S. A. 5ta edición. México. 2012. ISBN: 978-607-481-854-3.

[34] L. Blanco Encinosa. "La informática en la dirección de empresas". Félix Varela. La Habana, Cuba. 2011. ISBN: 978-959-07-1629-4.

[35] The Standish Group. "CHAOS Menifesto 2013. Think Big, Act Small”. Fecha de consulta: 10 de enero 2015. URL: http:// www.standishgroup.com 